\title{
Integrated Renewable Production of Sorbitol and Xylitol from Switchgrass
}

Guillermo Galán ${ }^{\mathrm{a}}$, Mariano Martín ${ }^{\mathrm{a}^{*}}$, Ignacio E. Grossmann ${ }^{\mathrm{b}}$.

${ }^{a}$ Department of Chemical Engineering. University of Salamanca. Plz Caidos 1-5, 37008, Salamanca, Spain

${ }^{b}$ Department of Chemical Engineering. Carnegie Mellon University, 5000 Forbes Ave, 15213, Pittsburgh PA, USA

mraiano.m3@usal

Supplementary Material

AFEX pretreatment

Table S1.- Range of operating variables for dilute acid pretreatment

\begin{tabular}{|l|c|c|}
\cline { 2 - 3 } \multicolumn{1}{c|}{} & Lower bound & Upper bound \\
\hline $\mathrm{T}\left({ }^{\circ} \mathrm{C}\right)$ & 90 & 180 \\
\hline Ammonia added $\left(\mathrm{g} \cdot \mathrm{g}^{-1}\right)$ & 0.5 & 2 \\
\hline Water added $\left(\mathrm{g} \cdot \mathrm{g}^{-1}\right)$ & 0.5 & 2 \\
\hline Residence time $(\mathrm{min})$ & 5 & 30 \\
\hline
\end{tabular}

Yield $=0.01 \cdot\left(-88.7919+26.5272 \cdot\right.$ amonia _ ratio $-13.6733 \cdot$ water_pret $+1.6561 \cdot \mathrm{T} \_$afex + $3.6793 \cdot$ time $\_$pret $-4.4631 \cdot$ amonia $_{-}$ratio $^{2}-0.0057 \cdot \mathrm{T}_{-}$afex $^{2}$

$-0.0270 \cdot$ time_pret ${ }^{2}-0.4064 \cdot$ amonia_ratio $\cdot$ time_pret $+0.1239 \cdot$ water_pret $\cdot \mathrm{T}$ _afex

$-0.0132 \cdot \mathrm{T}_{\text {_afex }} \cdot \mathrm{time} \_$pret$)$;

$$
\mathrm{Q}_{(\mathrm{HX} 2)}=\sum_{j} \mathrm{~m}_{(\mathrm{J}, \mathrm{HX} 2, \mathrm{Col1})} \cdot c_{p, j} \cdot\left(\mathrm{T}_{(\mathrm{HX} 2, \mathrm{Col} 1)}-\mathrm{T}_{(\mathrm{Valv} 1, \mathrm{HX} 2)}\right)
$$

Where: 


$$
\begin{aligned}
& \mathrm{T}_{(\mathrm{HX} 2, \mathrm{Coll})}=a_{\left(\mathrm{NH}_{3} / \text { Water }\right)} \cdot P^{2}+b_{\left(\mathrm{NH}_{3} / \text { Water }\right)} \cdot P+c_{\left(\mathrm{NH}_{3} / \text { Water }\right)} \\
& a_{\left(\mathrm{NH}_{3} / \text { Water }\right)}=1.0152 \cdot\left(\operatorname{Load}_{\text {Ammonia_Water }}\right)^{2}-0.3996 \cdot\left(\operatorname{Load}_{\text {Ammonia_Water }}\right)-2.118 \\
& b_{\left(\mathrm{NH}_{3} / \text { Water }\right)}=-11.344\left(\operatorname{Load}_{\text {Ammonia_Water }}\right)^{2}+8.6088 \cdot\left(\operatorname{Load}_{\text {Ammonia_Water }}\right)+25.159 ; \\
& c_{\left(\mathrm{NH}_{3} / \text { Water }\right)}=68.912\left(\operatorname{Load}_{\text {Ammonia_Water }}\right)^{2}-174.79 \cdot\left(\operatorname{Load}_{\text {Ammonia_Water }}\right)+59.214
\end{aligned}
$$

And the purity

$$
\begin{aligned}
& \text { Purity }=a_{\left(\mathrm{NH}_{3} / \text { Water }\right)} \cdot P^{2}+b_{\left(\mathrm{NH}_{3} / \text { Water }\right)} \cdot P+c_{\left(\mathrm{NH}_{3} / \text { Water }\right)} \\
& a_{\left(\mathrm{NH}_{3} / \text { Water }\right)}=-0.5792 \cdot\left(\operatorname{Load}_{\text {Ammonia_Water }}\right)^{2}-0.9987 \cdot\left(\operatorname{Load}_{\text {Ammonia_Water }}\right)+1.48 \\
& b_{\left(\mathrm{NH}_{3} / \text { Water }\right)}=9.9096\left(\operatorname{Load}_{\text {Ammonia_Water }}\right)^{2}-0.3672 \cdot\left(\operatorname{Load}_{\text {Ammonia_Water }}\right)-12.319 \\
& c_{\left(\mathrm{NH}_{3} / \text { Water }\right)}=-81.468\left(\operatorname{Load}_{\text {Ammonia_Water }}\right)^{2}+127.72\left(\operatorname{Load}_{\text {Ammonia_Water }}\right)+56.404
\end{aligned}
$$

Purity<99;

Purity $>80$

And the recovery yield of ammonia is given by eq. (5)

$$
\text { Yield }=-0.1123 \cdot\left(\text { Load }_{\text {Ammonia_Water }_{\text {W }}}\right)^{2}+0.2055 \cdot\left(\operatorname{Load}_{\text {Ammonia_ }_{\text {Water }}}\right)+99.889 \text {; }
$$

Rend<99.99;

Rend>99.9;

The energy balance to the column is modeled using the following surrogate models for the condenser and the reboiler respectively $(\mathrm{kW})$ per:

$$
\begin{aligned}
& \mathrm{Q}_{\text {condenser }}=\left(4.8709 \cdot\left(\operatorname{Load}_{\text {AAmmonia_Water }}\right)+1.4989\right) \frac{f_{c(\text { water })}[\mathrm{kg} / \mathrm{s}]}{0.028} \\
& \mathrm{Q}_{\text {Reboiler }}=\left(9.4089 \cdot\left(\operatorname{Load}_{\text {AAmmonia_Water }}\right)+2.7523\right) \frac{f_{c(\text { water })}[\mathrm{kg} / \mathrm{s}]}{0.028}
\end{aligned}
$$

The exit temperatures of the column are computed as follows: 


$$
\begin{aligned}
& T_{(\text {Col } 1 \text { Mix } 2)}=a_{\left(\mathrm{NH}_{3} / \text { Water }\right)} \cdot P^{2}+b_{\left(\mathrm{NH}_{3} / \text { Water }\right)} \cdot P+c_{\left(\mathrm{NH}_{3} / \text { Water }\right)} \\
& a_{\left(\mathrm{NH}_{3} / \text { Water }\right)}=-15.315 \cdot\left(\operatorname{Load}_{\text {Ammonia_Water }}\right)^{2}+13.426 \cdot\left(\operatorname{Load}_{\text {Ammonia_Water }}\right)-4.9178 \\
& b_{\left(\mathrm{NH}_{3} / \text { Water }\right)}=127.73\left(\operatorname{Load}_{\text {Ammonia_Water }}\right)^{2}-103.7 \cdot\left(\operatorname{Load}_{\text {Ammonia_Water }}\right)+47.523 \\
& c_{\left(\mathrm{NH}_{3} / \text { Water }\right)}=-238.48\left(\operatorname{Load}_{\text {Ammonia_Water }}\right)^{2}+116.32\left(\operatorname{Load}_{\text {Ammonia_Water }}\right)+25.337 \\
& \mathrm{~T}_{(\mathrm{Col1, \text {Mix } 3 )}}=-1.8617 \cdot P^{2}+24.16 \cdot P+77.491
\end{aligned}
$$

\section{Dilute Acid pretreatment}

Table S2.- Range of operating variables for dilute acid pretreatment

\begin{tabular}{|l|c|c|}
\cline { 2 - 3 } \multicolumn{1}{c|}{} & Lower bound & Upper bound \\
\hline $\mathrm{T}\left({ }^{\circ} \mathrm{C}\right)$ & 140 & 180 \\
\hline Acid concentration $\left(\mathrm{g} \cdot \mathrm{g} \cdot{ }^{1}\right)$ & 0.005 & 0.02 \\
\hline Residence time $(\mathrm{min})$ & 1 & 80 \\
\hline Enzyme load $\left(\mathrm{g} \cdot \mathrm{g}^{-1}\right)$ & 0.0048 & 0.0966 \\
\hline
\end{tabular}

The yield of glucose is given by:

yield_cellu $=-0.00055171+0.00355819 \cdot \mathrm{T} \_$acid $+0.00067402 \cdot \mathrm{conc}$ _acid _mix + time_pret·0.00100531-enzyme_add $0.0394809-0.0186704 \cdot \mathrm{T}$ _acid·conc_acid_mix $+0.00043556 \cdot \mathrm{T} \_$acid·time_pret $+0.0002265 \cdot \mathrm{T} \_$acid·enzyme_add

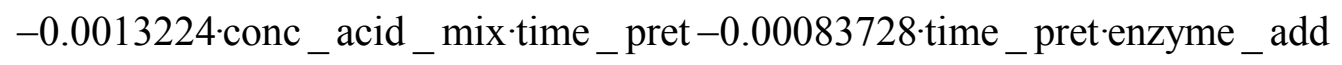
$+0.044353 \cdot$ conc_acid_mix enzyme_add $+0.000014412 \cdot \mathrm{T}_{-}$acid $^{2}$;

The yield of xylose is given by: 
yield_hemi $=-0.00015791-0.00056353 \cdot \mathrm{T}_{-}$acid $+0.000694361 \cdot \mathrm{conc}$ acid_mix $-0.00014507 \cdot$ time_pret - enzyme_add $0.01059248-0.02142606 \cdot \mathrm{T}$ _acid·conc_acid_mix $+0.000694055 \cdot \mathrm{T} \_$acid·time $\_$pret $+0.00013559 \cdot \mathrm{T}_{-}$acidenzyme_add $-0.00145712 \cdot$ conc_acid_mix $\cdot$ time_pret $+0.04769633 \cdot$ conc_acid_mix enzyme_add $-0.00138362 \cdot$ time_pretenzyme_add $+0.0000059419 \cdot \mathrm{T}_{-}$acid $^{2}$

\subsection{Xylitol and Sorbitol Production by fermentation pathway}

the best concentration. Figure 1 shows the representation of the fructose conversion data achieved by Zymomonas mobilis as a function of its concentration ${ }^{13}$. In this step the traces of glucose convert to gluconic acid are neglected. We assume the traces of glucose can be used as a nutrient by the bacteria.

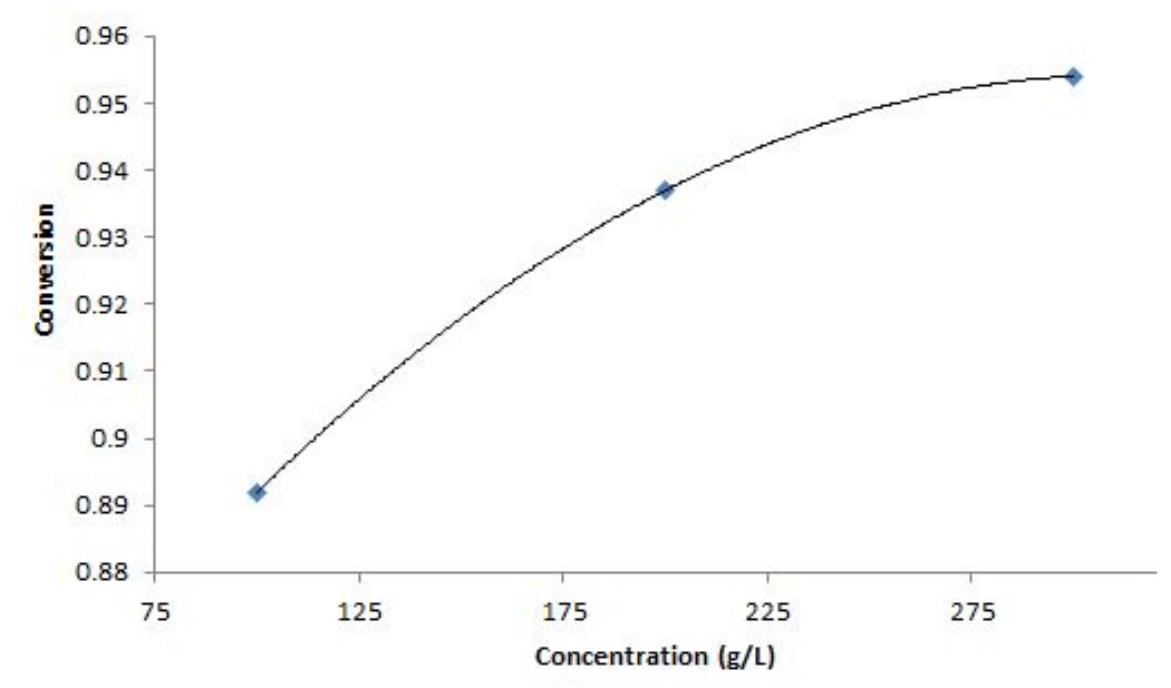

Figure S1. Fructose conversion with the concentration 


\section{Solubilities}

Xylose solubility

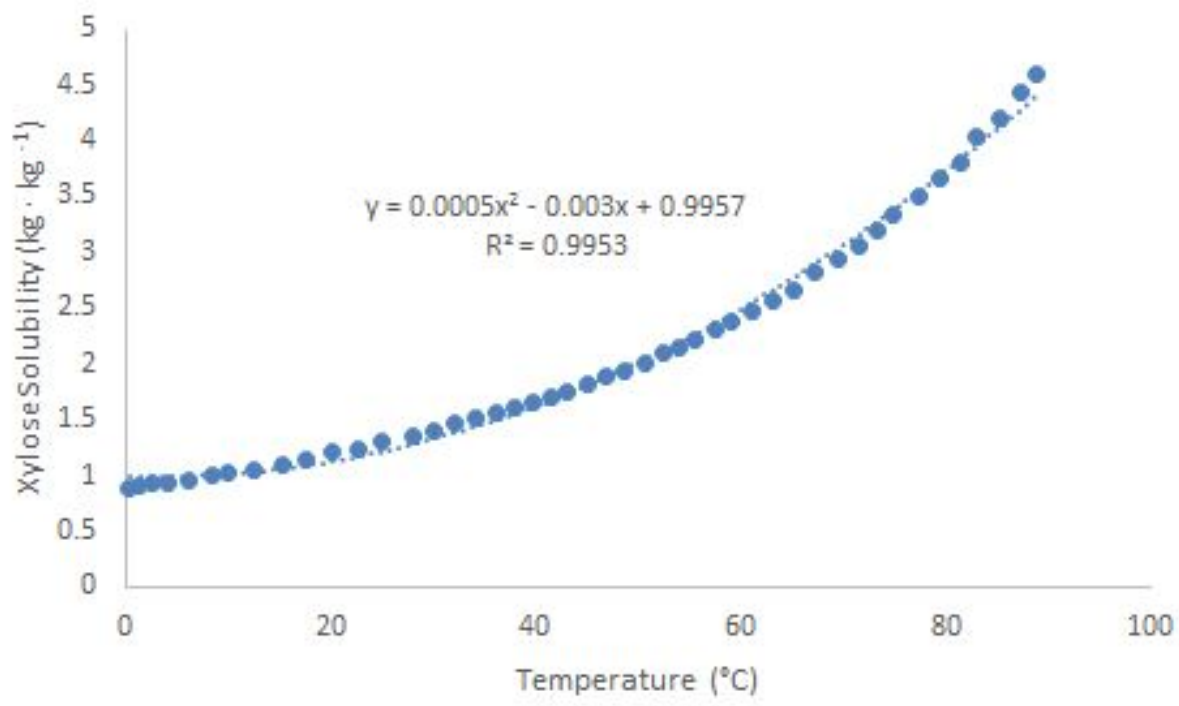

Figure S2. Xylose solubility

\section{Xylitol solubility}

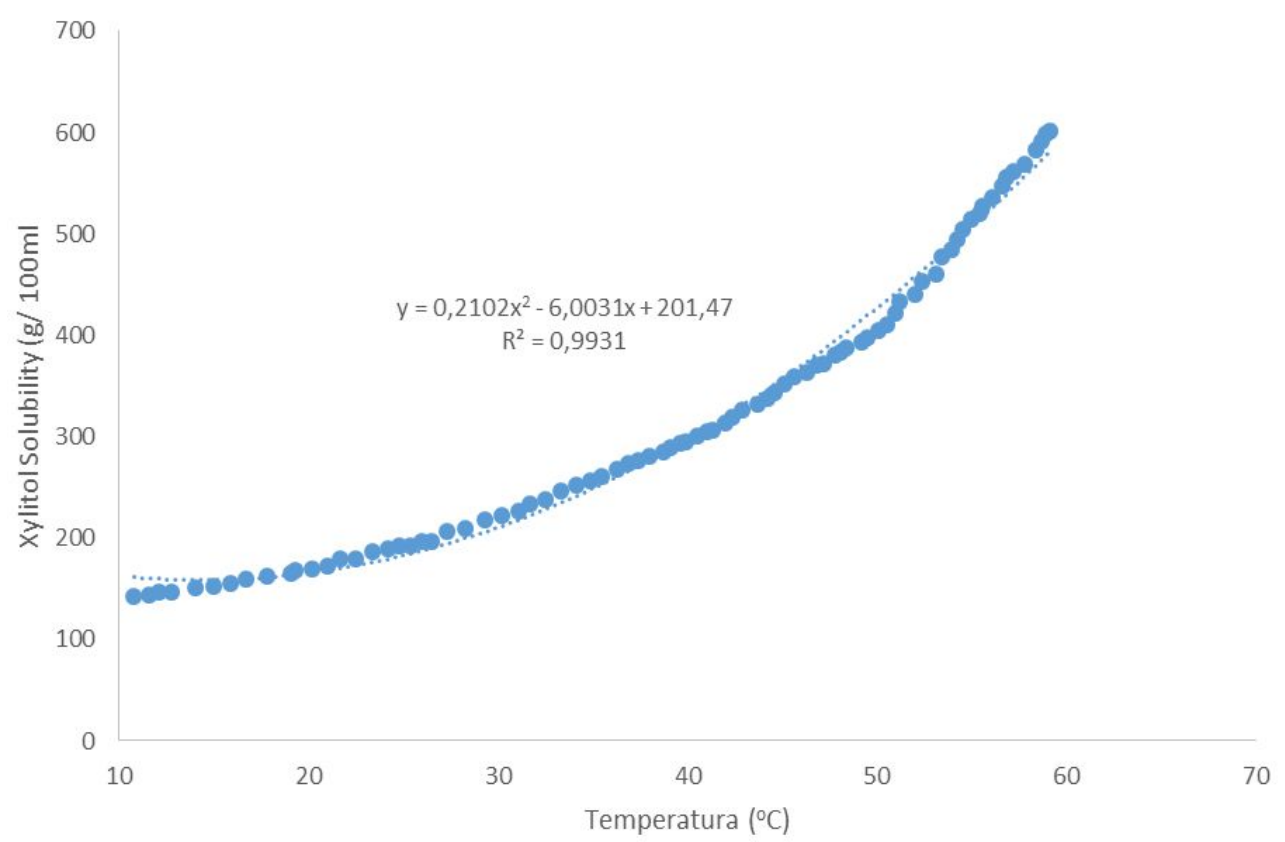

Figure S3. Xylitol solubility 


\section{Fructose solubility}

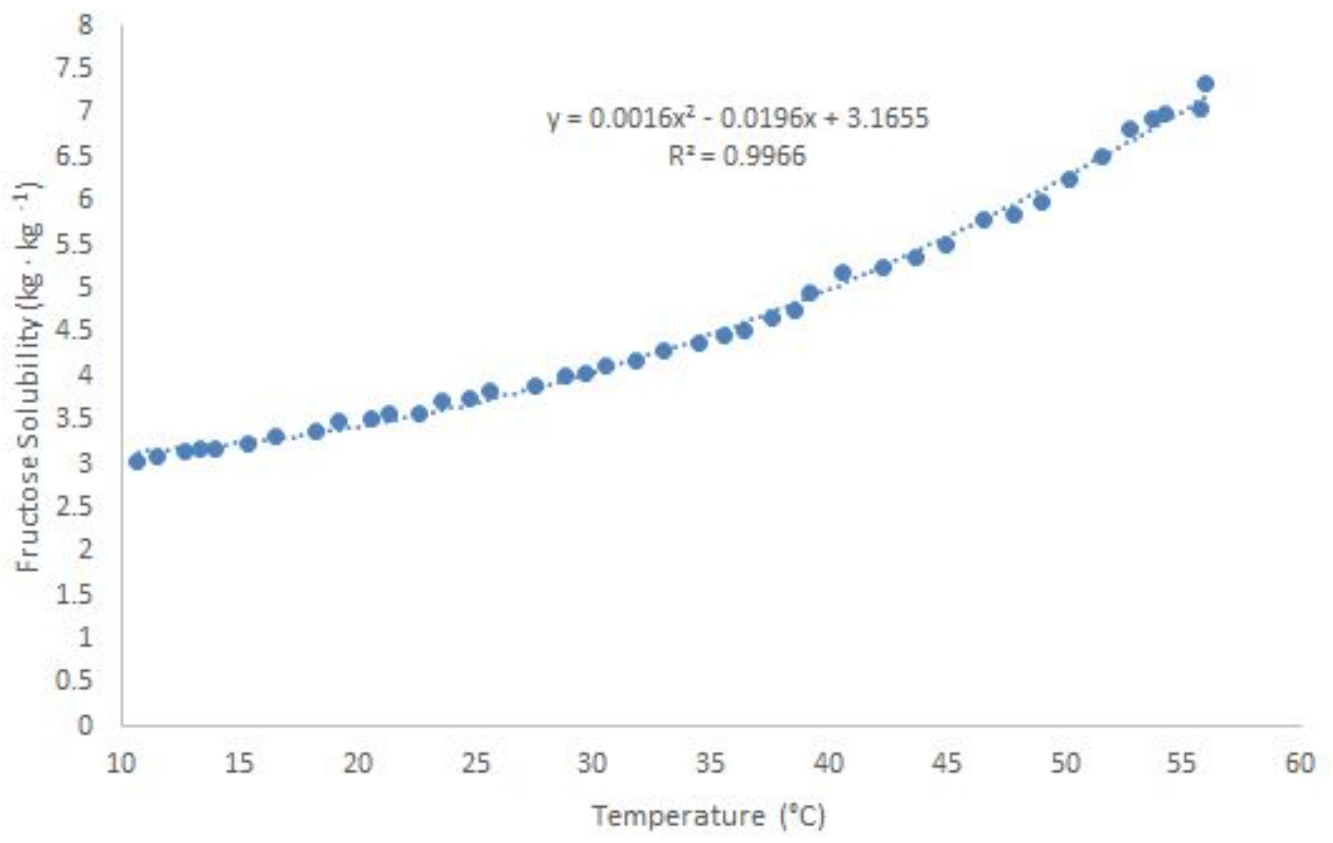

Figure S4. Fructose solubility

\section{Sorbitol solubility}

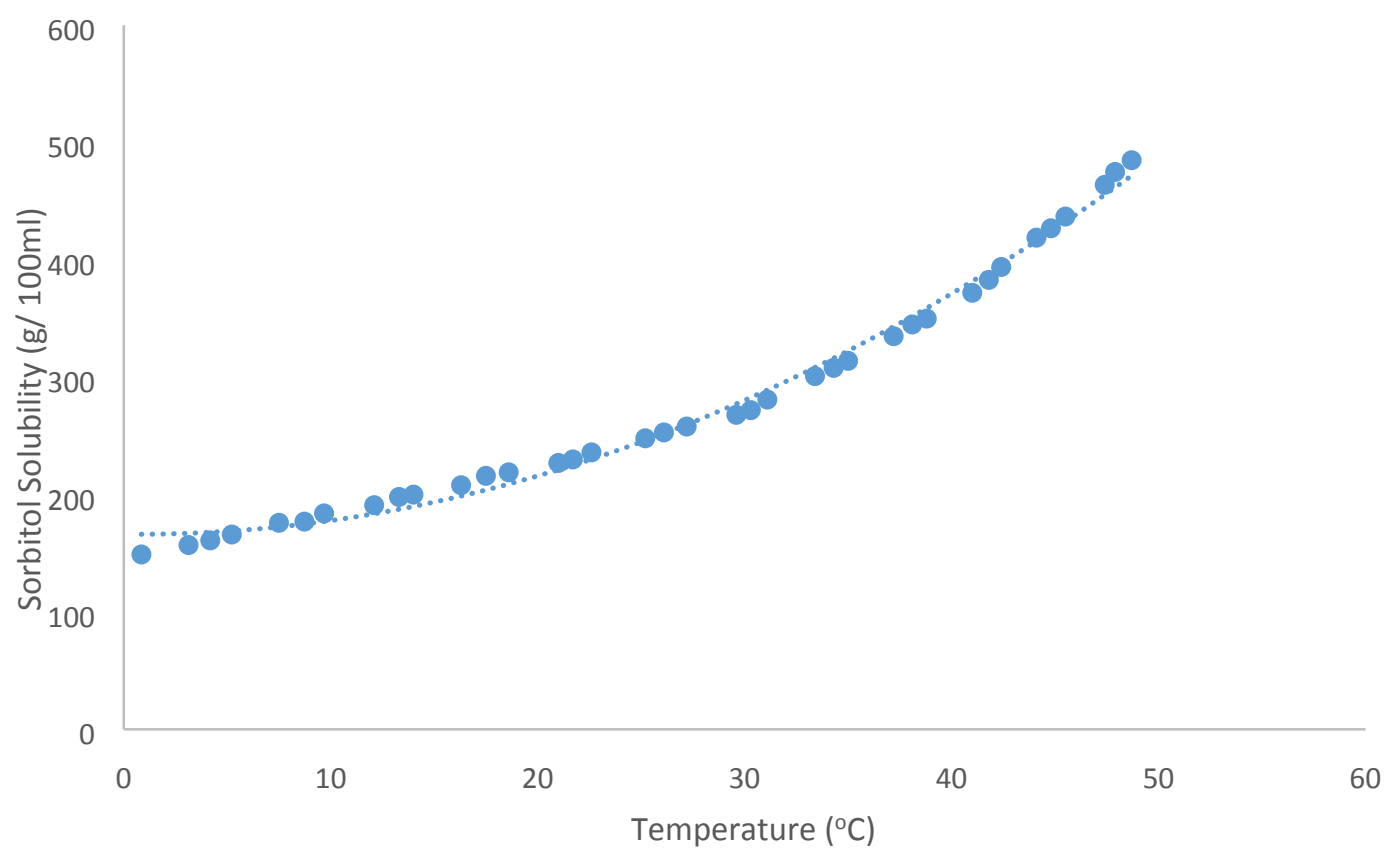

Figure S5. Sorbitol solubility 
Xylitol catalysis model
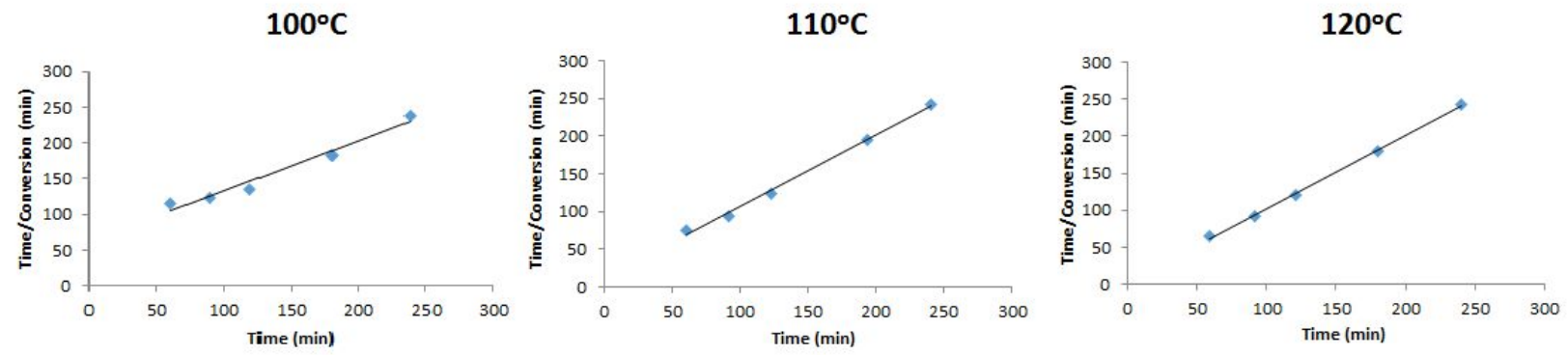

$100^{\circ} \mathrm{C}$

$110^{\circ} \mathrm{C}$

$120^{\circ} \mathrm{C}$
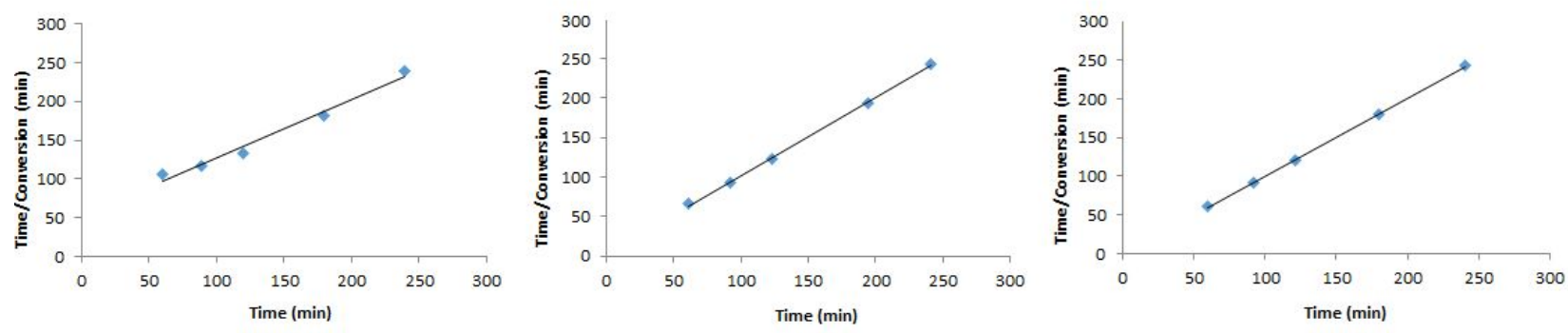

$100^{\circ} \mathrm{C}$

$110^{\circ} \mathrm{C}$
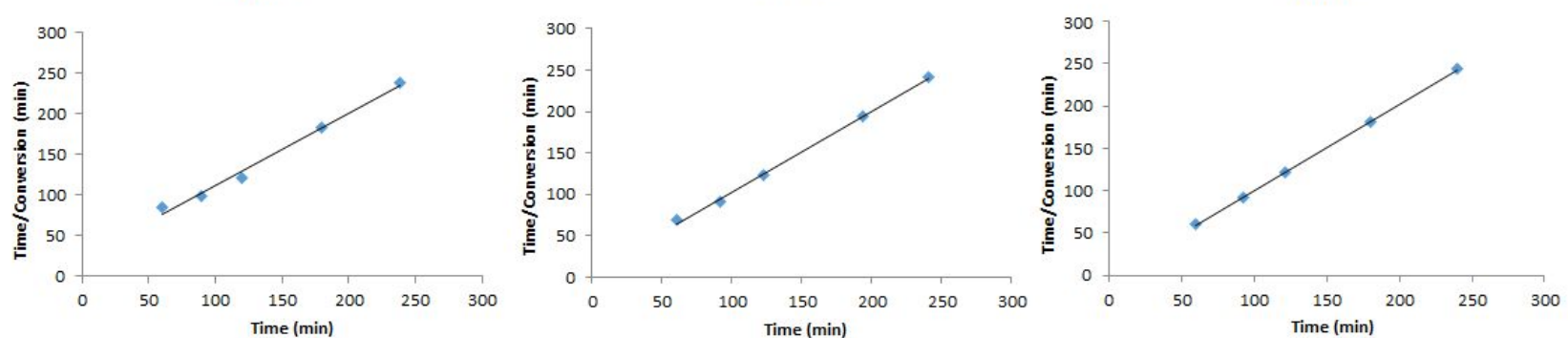

Figure S6. Linearization for 40,50 and 60 bar 


\section{Parameters fitting}

\section{Parameter $\mathrm{d}$ for $\mathbf{4 0}$ bar}

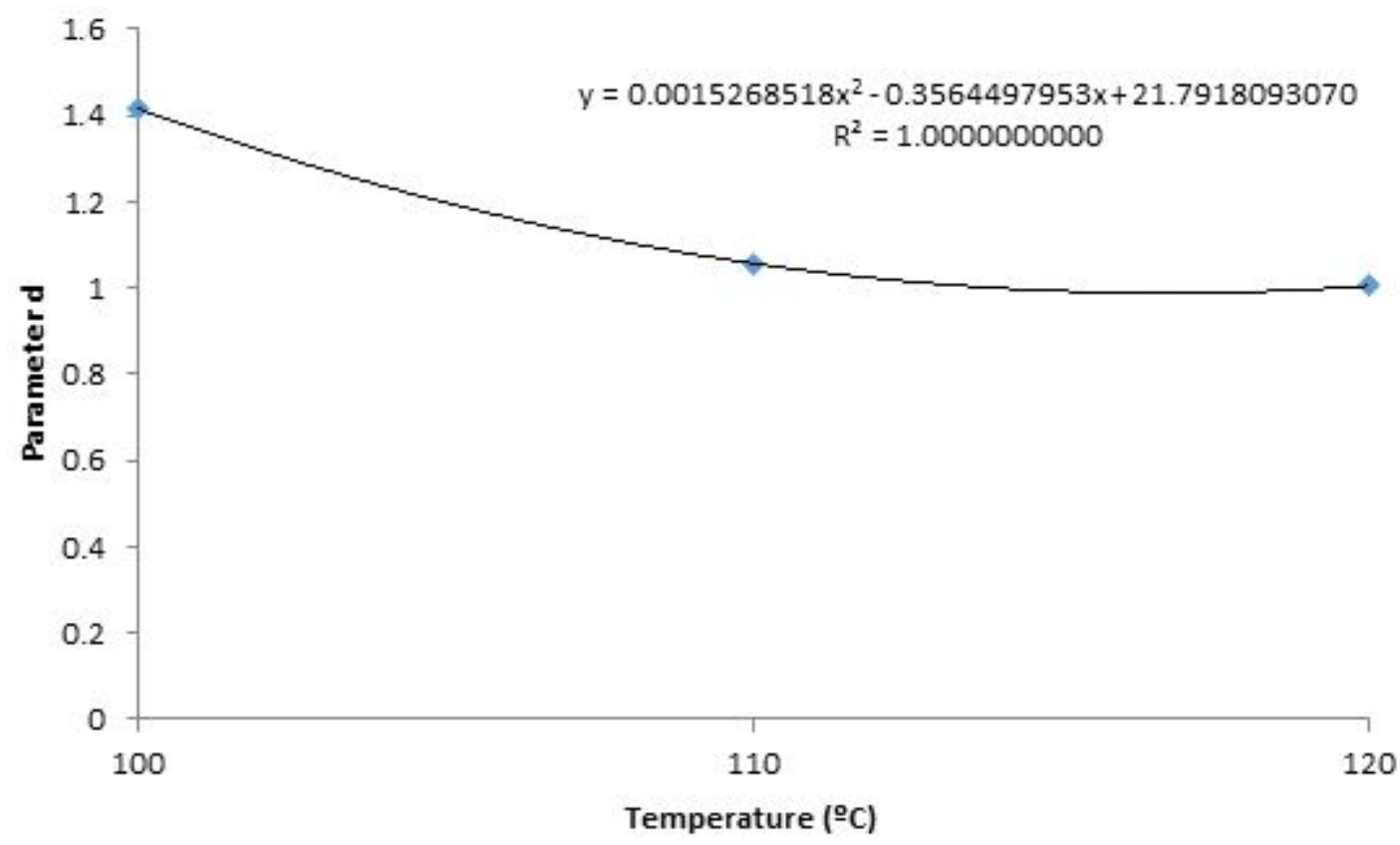

Figure S7. Parameter $d$ for 40 bar with the temperature

\section{Parameter $\mathrm{d}$ for $\mathbf{5 0}$ bar}

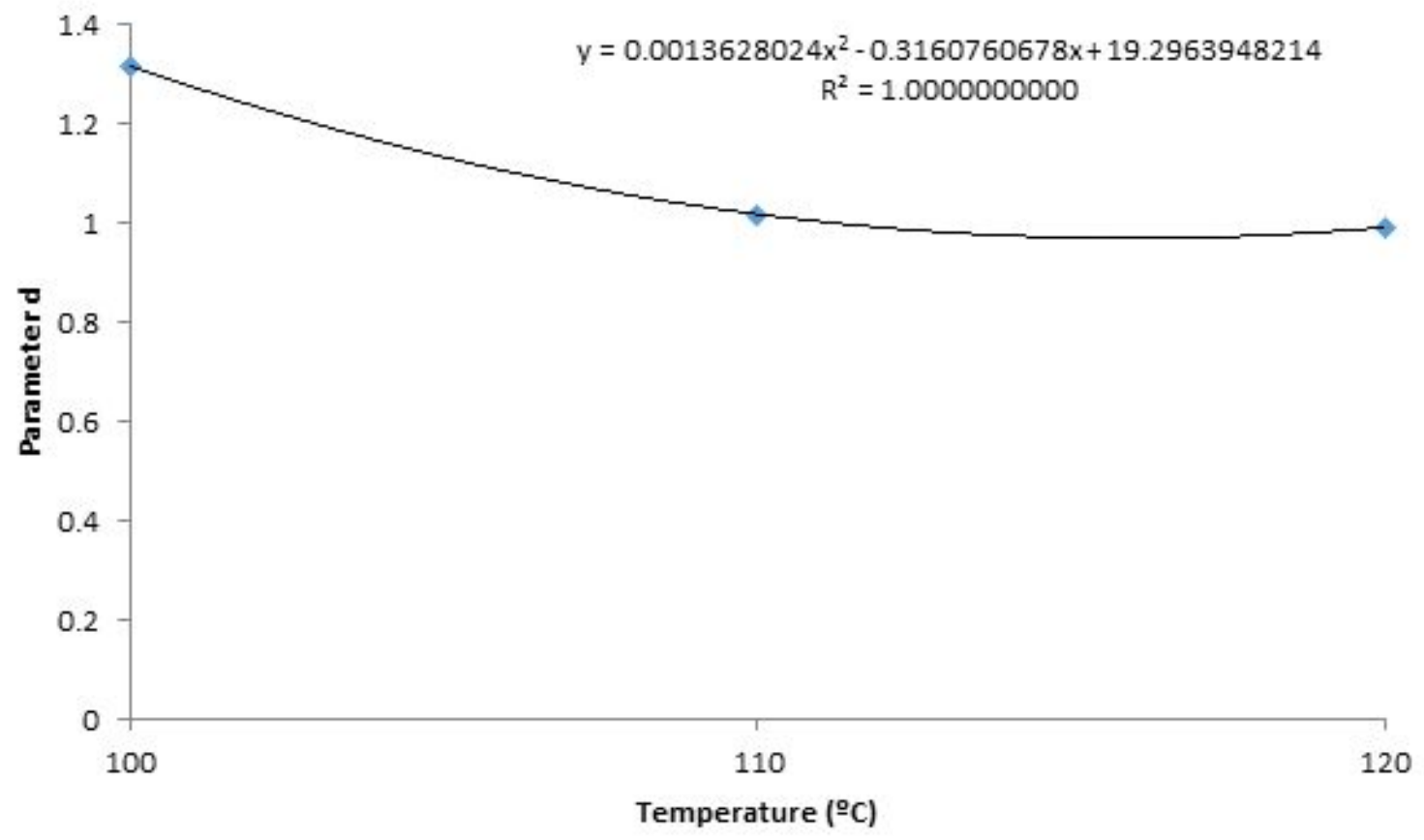

Figure S8. Parameter $d$ for 50 bar with the temperature 


\section{Parameter $\mathrm{d}$ for $\mathbf{6 0}$ bar}

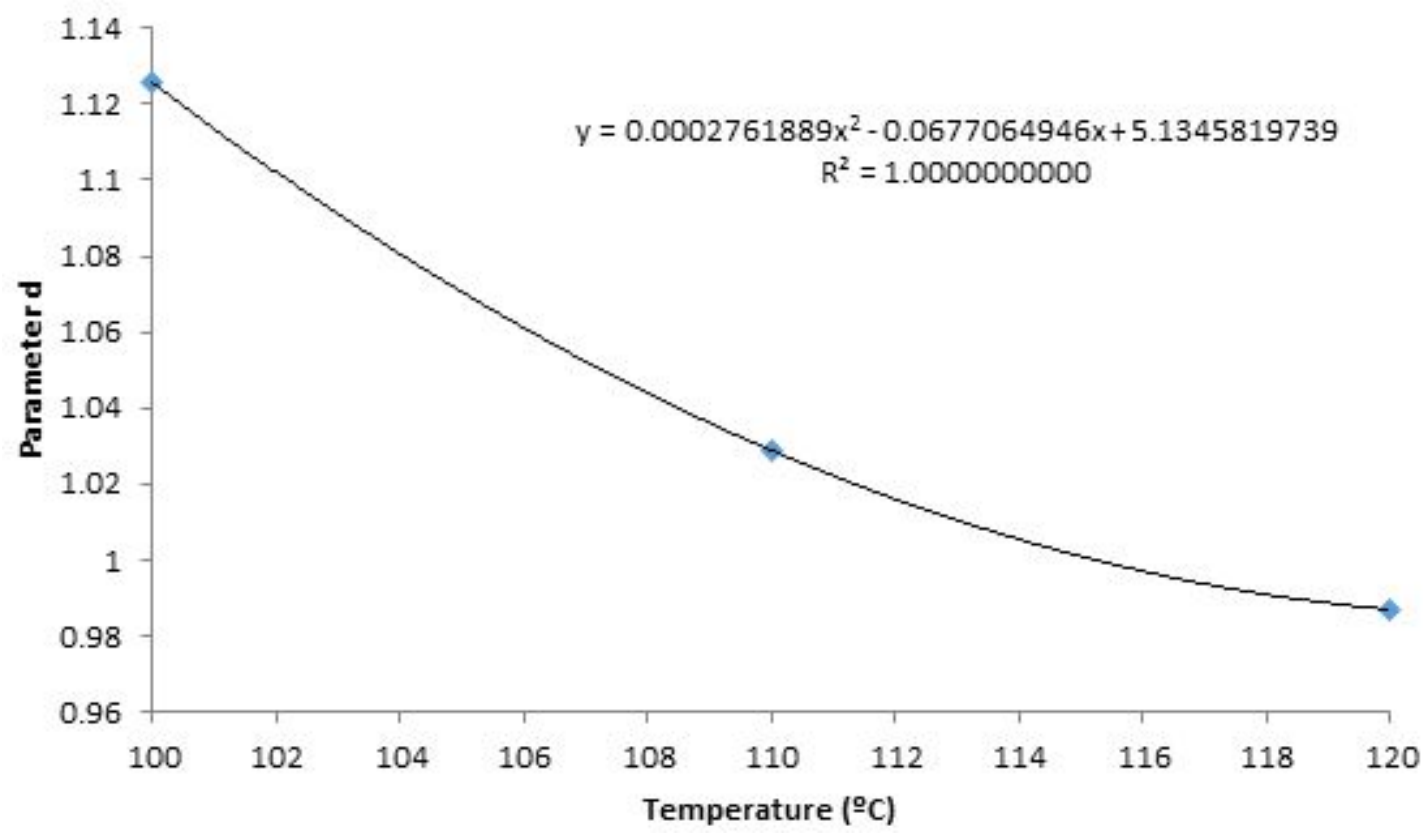

Figure S9. Parameter $d$ for 60 bar with the temperature

\section{Parameter e for $\mathbf{4 0}$ bar}

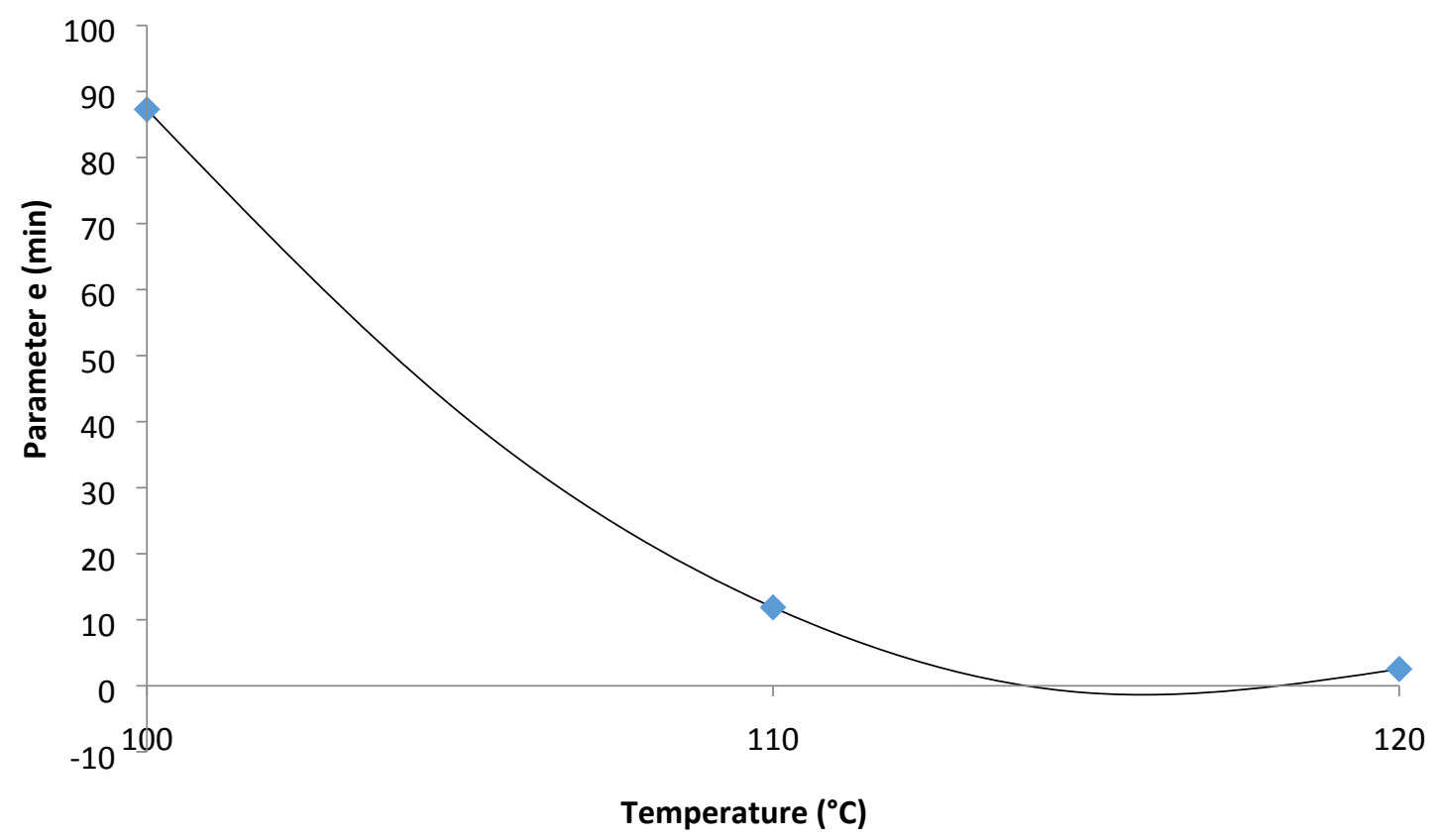

Figure S10. Parameter e for 40 bar with the temperature 
Parameter e for 50 bar

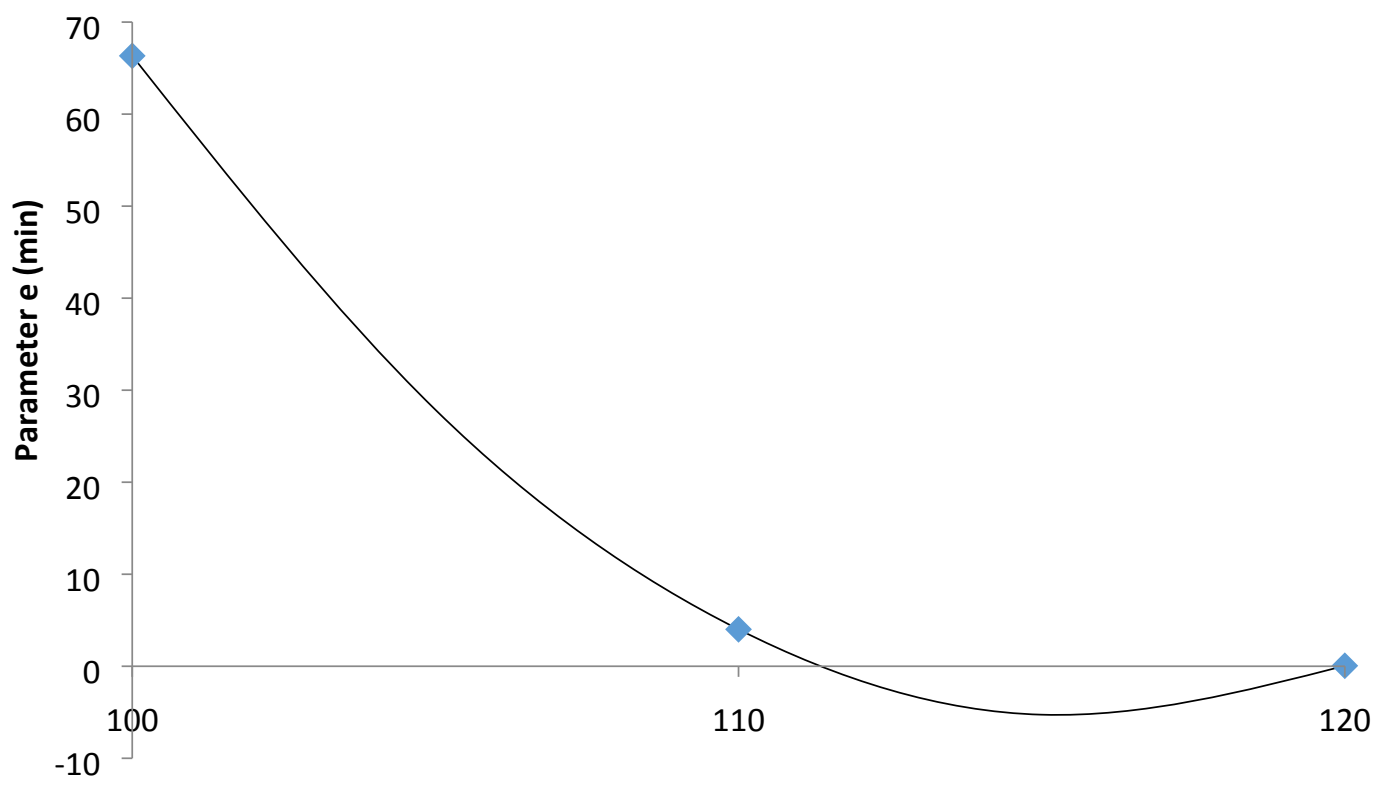

Temperature $\left({ }^{\circ} \mathrm{C}\right)$

Figure S11. Parameter e for 50 bar with the temperature

\section{Parameter e for 60 bar}

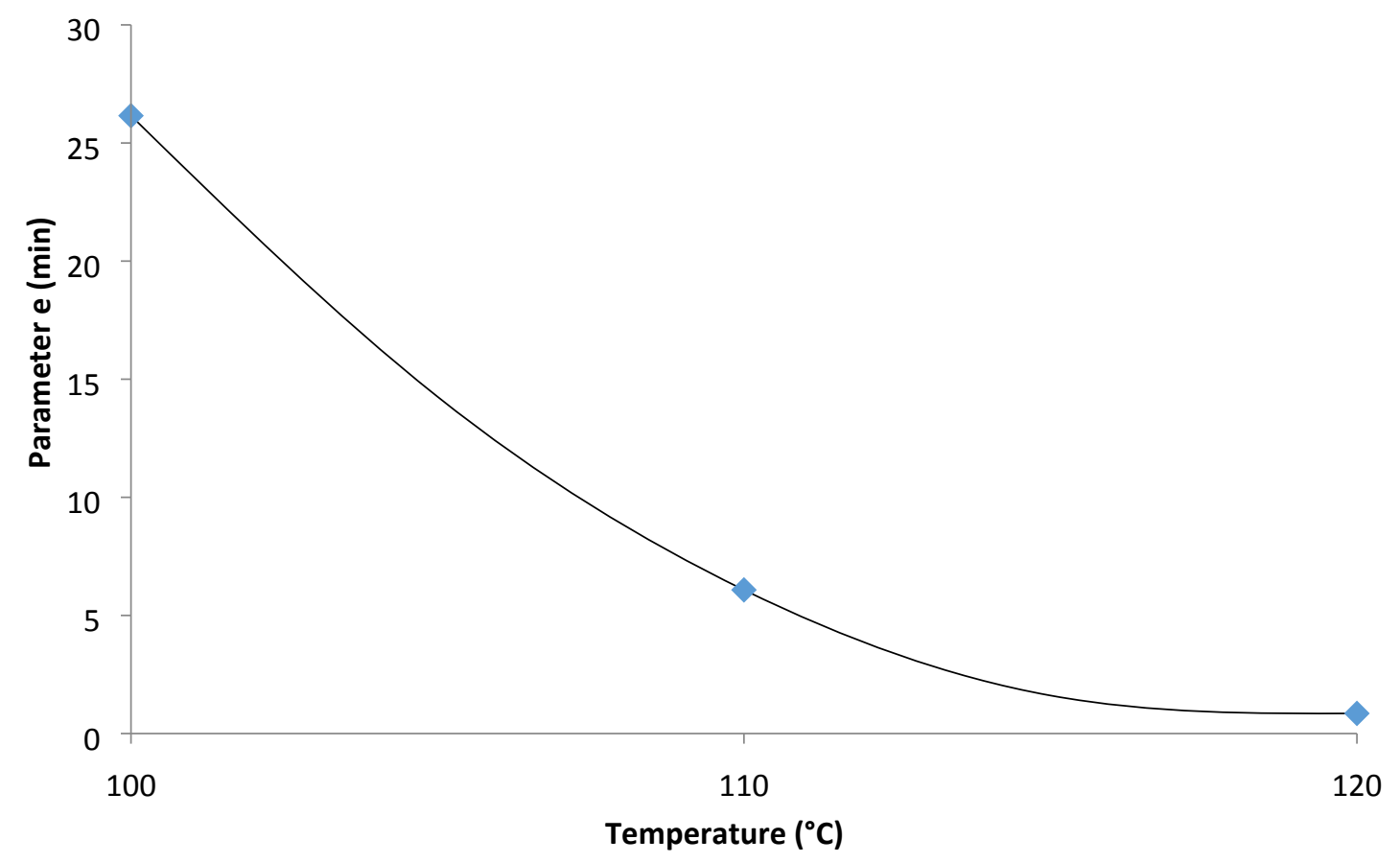

Figure S12. Parameter e for 60 bar with the temperature 


\section{Evaporators modeling}

$H_{L}=\sum l_{i} \cdot\left(\Delta \mathrm{H}_{\text {formi }}+\int_{T_{\text {ref }}}^{T} c_{p i} \cdot d T\right)$

Where $L=\sum l_{i}$

The steam used in the first effect, HS, is a saturated steam.

$H_{S}=S \cdot\left(\Delta \mathrm{H}_{\text {form Wa(liq) }}+\int_{T_{r f}}^{T_{e b}} c_{p, l i q} \cdot d T+\lambda\left(\mathrm{T}_{e b}\right)\right)$

Hs is the enthalpy of the condensed steam and it"s calculated as follows:

$H_{s}=S \cdot\left(\Delta \mathrm{H}_{\text {form Wa(liq) }}+\int_{T_{r f}}^{T_{c b}} c_{p, l i q} \cdot d T\right)$ 


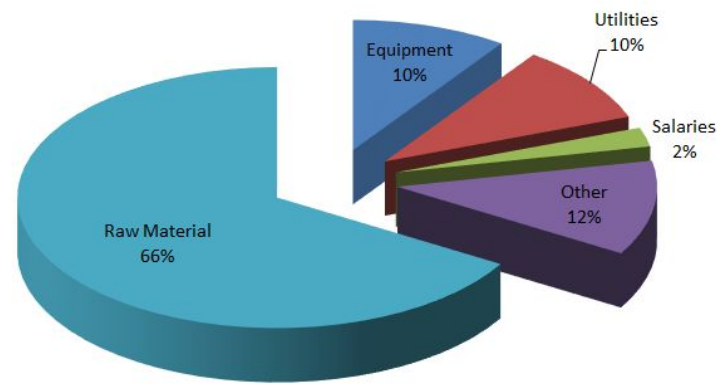

b)

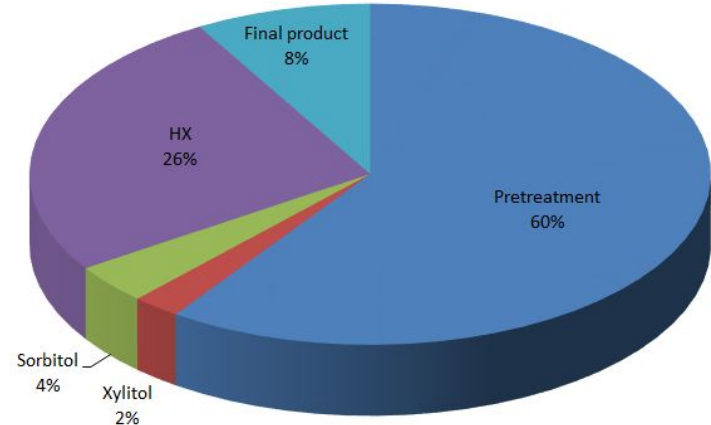

Figure S13. Dilute Acid- Catalytic Hydrogenation Corn Stover

a)

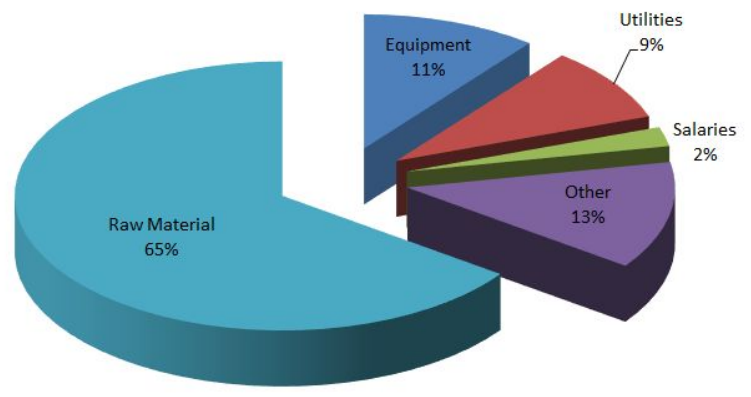

b)

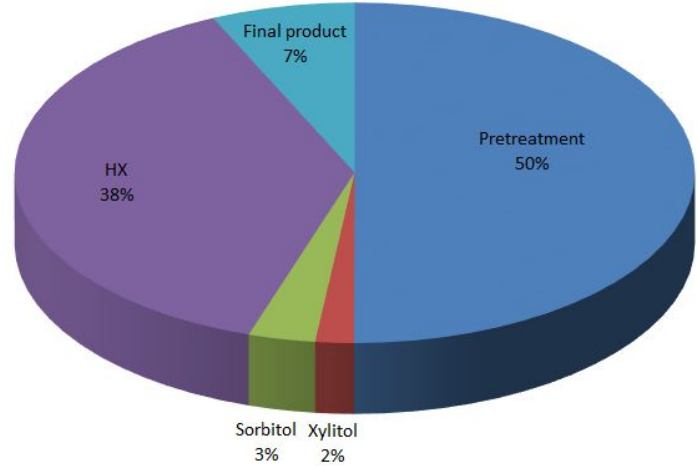

Figure S14. Dilute Acid- Catalytic Hydrogenation Birch

a)

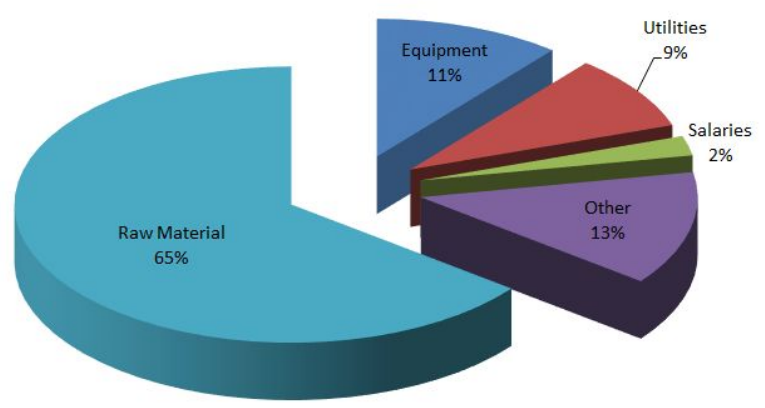

b)

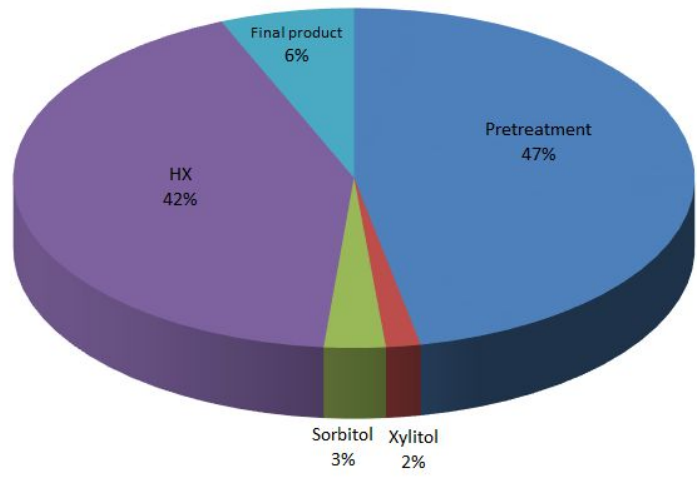

Figure S15. Dilute Acid- Catalytic Hydrogenation Pine 
a)

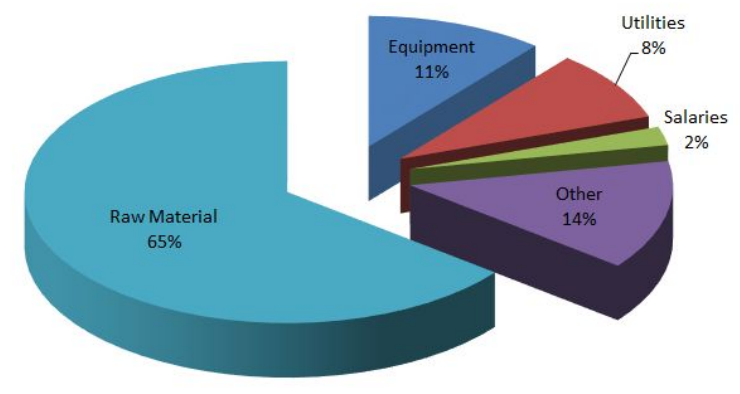

b)

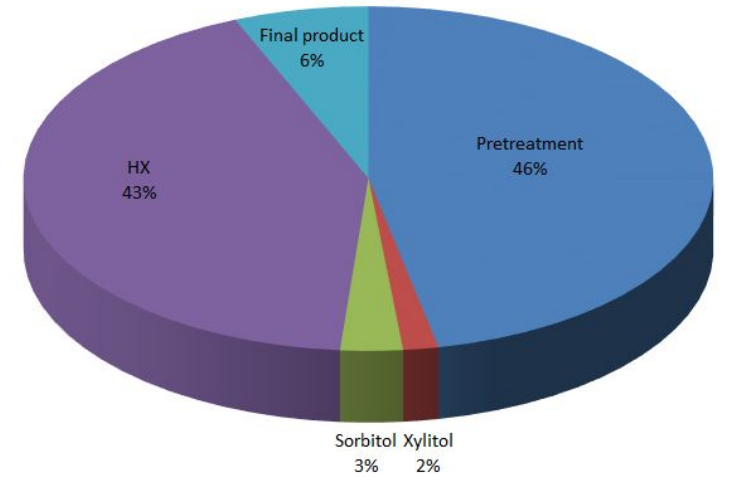

Figure S16. Dilute Acid- Catalytic Hydrogenation Spruce

a)

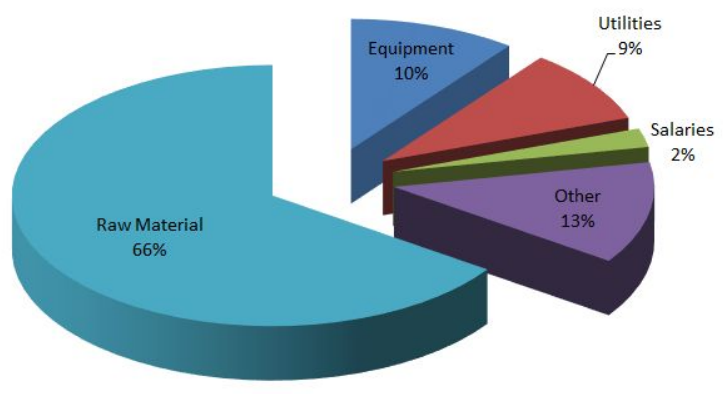

b)

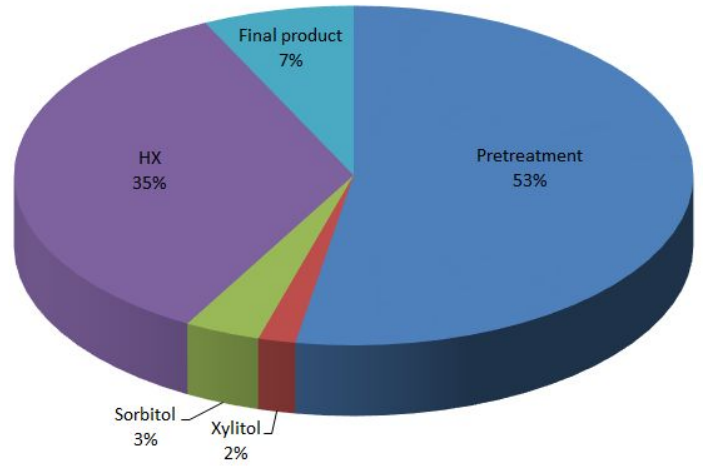

Figure S17. Dilute Acid- Catalytic Hydrogenation Poplar

a)

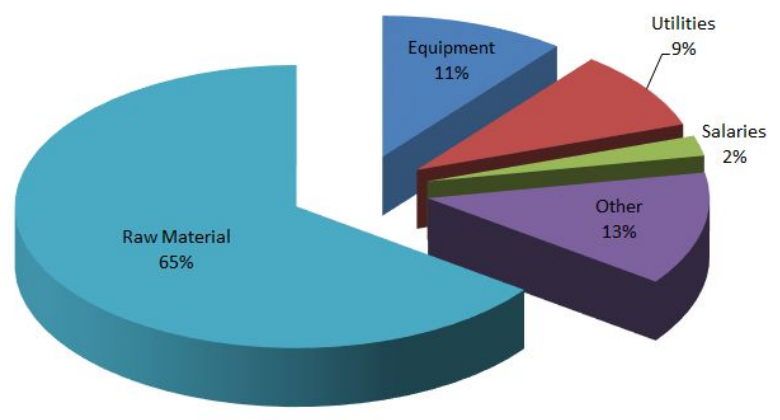

b)

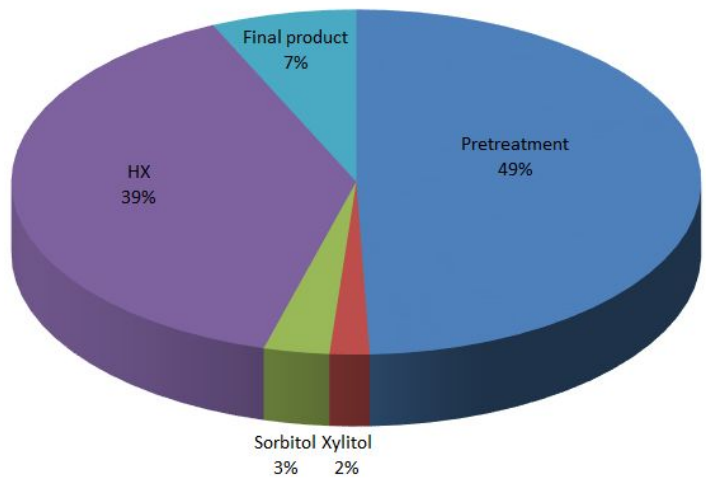

Figure S18. Dilute Acid- Catalytic Hydrogenation Sugarcane Bagasse 
a)

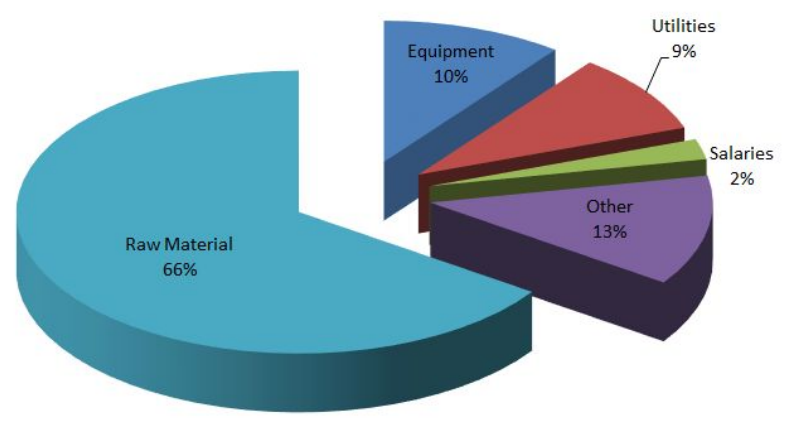

b)

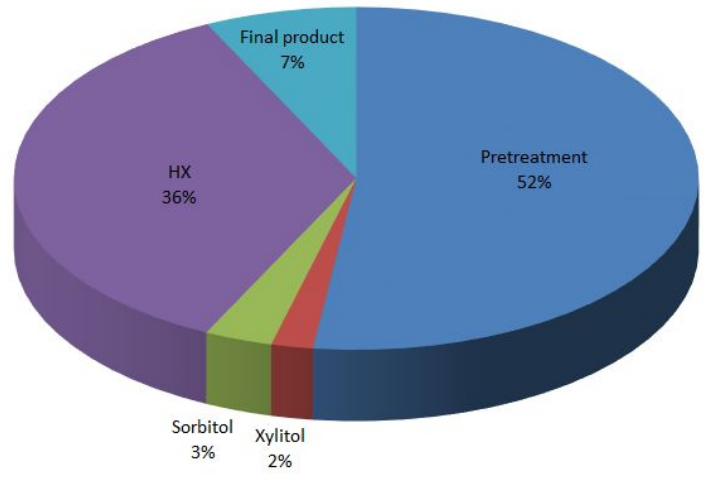

Figure S19. Dilute Acid- Catalytic Hydrogenation Wheat Straw 